\title{
Self-generated Magnetic Fields in Blast-wave Driven Rayleigh-Taylor Experiments
}

\author{
Markus Flaig ${ }^{\mathrm{a}, *}$, Tomasz Plewa ${ }^{\mathrm{a}}$ \\ ${ }^{a}$ Florida State University, USA
}

\begin{abstract}
We study the effect of self-generated magnetic fields in two-dimensional computer models of blast-wave driven high-energy density Rayleigh-Taylor instability (RTI) experiments. Previous works $[1,2]$ suggested that such fields have the potential to influence the RTI morphology and mixing. When neglecting the friction force between electrons and ions, we do indeed find that dynamically important $\left(\beta \lesssim 10^{3}\right)$ magnetic fields are generated. However, in the more realistic case where the friction force is accounted for, the resulting fields are much weaker, $\beta \gtrsim 10^{5}$, and can no longer influence the dynamics of the system. Although we find no evidence for dynamically important magnetic fields being created in the two-dimensional case studied here, the situation might be different in a three-dimensional setup, which will be adressed in a future study.
\end{abstract}

Keywords: hydrodynamics, hydrodynamic instabilities, laboratory astrophysics, Rayleigh-Taylor, HEDP laboratory experiments

\section{Introduction}

Observational as well as theoretical studies [3] have shown that during a supernova explosion, various fluid-dynamical instabilities (such as the Rayleigh-Taylor instability [RTI, see refs. 4,5$]$ ) lead to mixing between the material shells of the exploding star. Understanding the observed mixing has been proved difficult, and in many cases numerical simulations of supernova explosions do not match observational results [see 6, and references therein].

High-energy density laboratory astrophysics experiments [7, $8,9]$ which reproduce the physical conditions in the exploding star can be used as a tool to help understand the physical processes responsible for the mixing. In these experiments, one uses powerful lasers to drive a shock wave through a target, which is composed of two or more layers of materials with different densities. The mixing that occurs between the material layers can be observed via x-ray radiography. Although there is a huge difference in scale between such experiments and a supernova, a direct connection between the two systems can be made by employing similarity scaling [10], provided that viscous and radiative effects can be neglected.

The first experiments of this type were performed on the NOVA laser [11, 12]. Later work by Kuranz and her collaborators $[9,13,14]$ continued on the OMEGA laser using planar targets. In one particular case [14], the experimental radiographic images indicated the presence of dense plasma protruding from the tips of the RTI spikes toward the shock front. Subsequent computational studies of the experiment consistently failed to reproduce these features $[9,15,16]$. It has been suggested [1] that the reason for the discrepancy between experiment and simulations might be due to magnetic fields generated

${ }^{*}$ Corresponding Author.

E-mail address: mflaig@fsu.edu (M. Flaig) by the Biermann battery effect, which was not included in the numerical simulations.

Self-generated magnetic fields have been measured in an ablative RTI experiments, both during the linear [17] and nonlinear [18] growth phase of the instability. Numerical simulations indicate that magnetic fields can be dynamically important in the context of inertially confined fusion [2].

The aim of the present study is to investigate to what extent self-generated magnetic fields might have influenced the results of the Kuranz et al. experiment and if such fields might be important in other (similar) experiments. We discuss the results of numerical simulations of blast-wave driven RTI experiments based on the extended magnetohydrodynamics (MHD) formulation according to Braginskii [19], which includes the Biermann battery term. We consider two sets of parameters, where the first set corresponds to the Kuranz et al. experiment, and the second set corresponds to a recently proposed experiment [6] for the National Ignition Facility (NIF) laser.

\section{Model}

\subsection{Equations solved}

For the numerical simulations presented in this paper, we use the PROTEUS code [15]. PROTEUS is an extended version of the FLASH code [20], which includes additional physics for the modelling of anisotropic thermal conduction and magnetic field generation. Our work builds on the study done by [15], which considered the classical Rayleigh-Taylor problem of a heavy fluid sitting atop of a light fluid in a gravitational field. While their study was done in the context of the Braginskii formulation, it neglected the friction force between electrons and ions. In the present work, we consider blast-wave driven systems with parameters relevant to laser-driven shock experiments and also take into account the dissipation of the magnetic field due to the electron-ion friction force. 
We consider the following set of extended MHD equations in the single fluid approximation:

$$
\begin{aligned}
& \frac{\partial \rho}{\partial t}+\boldsymbol{\nabla} \cdot(\rho \boldsymbol{v})=0, \\
& \frac{\partial \rho \boldsymbol{v}}{\partial t}+\boldsymbol{\nabla} \cdot\left(\rho \boldsymbol{v} \boldsymbol{v}-\frac{\boldsymbol{B} \boldsymbol{B}}{4 \pi}\right)+\boldsymbol{\nabla} P=0, \\
& \frac{\partial E}{\partial t}+\boldsymbol{\nabla} \cdot\left[(E+P) \boldsymbol{v}-\frac{\boldsymbol{B} \boldsymbol{B}}{4 \pi} \cdot \boldsymbol{v}+\frac{\boldsymbol{B}}{4 \pi} \times \boldsymbol{S}_{\mathrm{GenOhm}}\right]=\boldsymbol{\nabla} \cdot \boldsymbol{q}, \\
& \frac{\partial \boldsymbol{B}}{\partial t}=\boldsymbol{\nabla} \times\left(\boldsymbol{v} \times \boldsymbol{B}+\boldsymbol{S}_{\mathrm{GenOhm}}\right) ;
\end{aligned}
$$

where

$$
\boldsymbol{S}_{\mathrm{GenOhm}}=\frac{c}{e n_{\mathrm{e}}}\left[k_{\mathrm{B}} \boldsymbol{\nabla}\left(n_{\mathrm{e}} T\right)-\frac{(\boldsymbol{\nabla} \times \boldsymbol{B}) \times \boldsymbol{B}}{4 \pi}-\boldsymbol{R}_{u}-\boldsymbol{R}_{T}\right] .
$$

In the above equations, $\rho$ denotes the plasma density, $v$ the velocity, $E$ the total energy density, $P$ the total pressure, $\boldsymbol{B}$ the magnetic field, $n_{\mathrm{e}}$ the number density of the electrons and $T$ the temperature, where we assume that ion and electron temperatures are equal $\left(T=T_{\mathrm{e}}=T_{\mathrm{i}}\right)$. The above equations are supplemented by an ideal gas equation of state with adiabiatic index $\gamma=5 / 3$.

We assume a plasma composed of multiple species. The ion number density is $n_{\mathrm{i}}=\rho N_{\mathrm{A}} / A$, with $\bar{A}$ the average atomic mass and $N_{\mathrm{A}}$ the Avogadro constant. In order to calculate the electron number density $n_{\mathrm{e}}$, we first compute the (average) plasma charge, $\bar{Z}$, using the Thomas-Fermi equation of state [21]. The corresponding electron number density is then $n_{\mathrm{e}}=\bar{Z} n_{\mathrm{i}}$.

The term on the right hand side of Eq. (1c) describes anisotropic thermal conduction. The heat flux vector $\boldsymbol{q}$ is given by:

$$
\boldsymbol{q}=-\kappa_{1}(\boldsymbol{b} \cdot \boldsymbol{\nabla} T) \boldsymbol{b}-\kappa_{2} \boldsymbol{b} \times(\boldsymbol{\nabla} T \times \boldsymbol{b})-\kappa_{3} \boldsymbol{b} \times \boldsymbol{\nabla} T,
$$

where $\boldsymbol{b}=\boldsymbol{B} / \boldsymbol{B}$ is a unit vector in the direction of the magnetic field. The coefficients $\kappa_{i}$ are of order $n_{\mathrm{e}} k_{\mathrm{B}} T \tau_{\mathrm{e}} / m_{\mathrm{e}}$, where $\tau_{\mathrm{e}}$ is the electron-ion scattering time [19]. They are related to the coefficients calculated in Braginskii's paper [19] as follows:

$$
\begin{aligned}
& \kappa_{1}=\kappa_{\|}^{\mathrm{e}}+\kappa_{\|}^{\mathrm{i}}, \\
& \kappa_{2}=\kappa_{\perp}^{\mathrm{e}}+\kappa_{\perp}^{\mathrm{i}}, \\
& \kappa_{3}=\kappa_{\wedge}^{\mathrm{e}}-\kappa_{\wedge}^{\mathrm{i}} .
\end{aligned}
$$

In Eq. (1e), the last two terms in the square brackets are the electron-ion friction force $\left(\boldsymbol{R}_{u}\right)$, and the thermal force $\left(\boldsymbol{R}_{T}\right)$, which are responsible for diffusing (and thereby destroying) the magnetic field. These two terms are defined by the following formulae:

$$
\begin{gathered}
\boldsymbol{R}_{u}=\alpha_{1}(\boldsymbol{b} \cdot \boldsymbol{J}) \boldsymbol{b}+\alpha_{2} \boldsymbol{b} \times(\boldsymbol{J} \times \boldsymbol{b})-\alpha_{3} \boldsymbol{b} \times \boldsymbol{J}, \\
\boldsymbol{R}_{T}=-\beta_{1}(\boldsymbol{b} \cdot \nabla T) \boldsymbol{b}-\beta_{2} \boldsymbol{b} \times(\boldsymbol{\nabla} \times \boldsymbol{b})-\beta_{3} \boldsymbol{b} \times \boldsymbol{\nabla} T ;
\end{gathered}
$$

where $\boldsymbol{J}=(c / 4 \pi) \boldsymbol{\nabla} \times \boldsymbol{B}$ is the current. The coefficients $\alpha_{i}$ and $\beta_{i}$ are of order $m_{\mathrm{e}} / e \tau_{\mathrm{e}}$ and $k_{\mathrm{B}} n_{\mathrm{e}}$, respectively [for details,

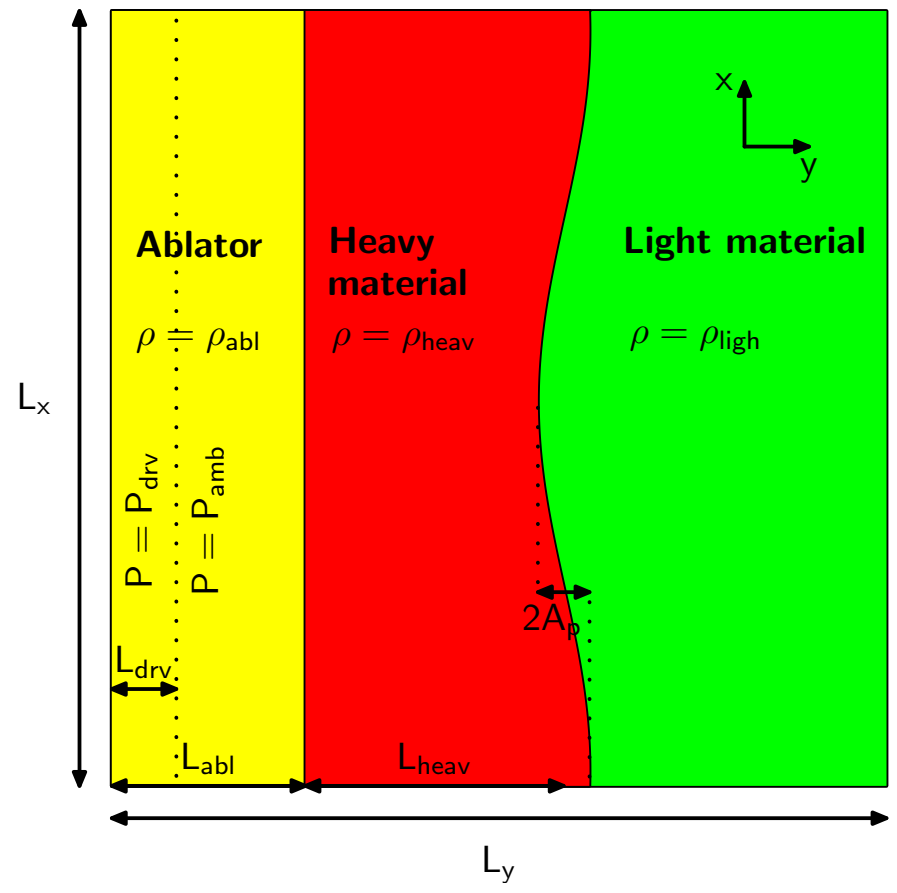

Figure 1: Sketch of the simulation setup. The target consists of a layer of heavy material and a layer of light material, with a sinusoidal perturbation of amplitude $A_{\mathrm{p}}$ on the interface. In addition to this, some of the simulations have an additional layer which corresponds to the ablator. Simulated is a section of the target with a width of one perturbation wavelength. In the region to the left of the dotted line (with width $L_{\mathrm{drv}}$ ), the pressure is set initially to a high value, corresponding to the pressure created by the laser drive.

see 19]. The first term inside the square brackets in Eq. (1e) is responsible for the Biermann battery effect. The change in the magnetic field due to this term can be written as

$$
\frac{\partial \boldsymbol{B}}{\partial t}=\frac{c k_{\mathrm{B}}}{e} \boldsymbol{\nabla} T_{\mathrm{e}} \times \nabla \ln n_{\mathrm{e}}
$$

i.e. magnetic fields are generated due to a thermally driven current whenever the electron temperature and electron density gradients are misaligned.

For the derivation of the above equations and further details, we point the reader to the original work by Braginskii [19].

In our simulations, we employ the unsplit staggered mesh scheme described in [22], which uses a constrained transport method that preserves the $\boldsymbol{\nabla} \cdot \boldsymbol{B}=0$ constraint to machine accuracy. For all simulations, we use the Roe Riemann solver and second order reconstruction. We do not consider time-step limiting due to whistler and Hall drift waves. Instead we set the CFL number to a small value $(\sim 0.01)$ to ensure stability.

\subsection{Initial conditions}

We perform two-dimensional Cartesian simulations of a single blast-wave driven Rayleigh-Taylor spike. For simplicity, our simulations do not include the heating due to the laser drive; instead, the simulations are initialized with a small region of very high pressure on the side of the target which in an experiment would be illuminated by the laser. The domain covers a rectangular region with dimensions $\left[-L_{x} / 2, L_{x} / 2\right] \times\left[0, L_{y}\right]$, 
where $L_{x}$ and $L_{y}$ correspond to the lateral and longitudinal domain size, respectively. The initial conditions are as follows:

$$
\begin{aligned}
& \rho(x, y)= \begin{cases}\rho_{\mathrm{abl}}, & y \leq L_{\mathrm{abl}} \\
\rho_{\mathrm{heav}}, & L_{\mathrm{abl}} \leq y \leq L_{\mathrm{abl}}+L_{\text {heav }}+\delta(x), \\
\rho_{\text {ligh }}, & \text { otherwise }\end{cases} \\
& P(x, y)=\left\{\begin{array}{ll}
P_{\mathrm{drv}}, & y \leq L_{\mathrm{drv}} \\
P_{\mathrm{amb}}, & \text { otherwise }
\end{array},\right. \\
& \boldsymbol{v}(x, y)=0,
\end{aligned}
$$$$
\boldsymbol{B}(x, y)=\boldsymbol{B}_{0} .
$$

Here, $\rho_{\mathrm{abl}}, \rho_{\text {heav }}$ and $\rho_{\text {ligh }}$ are the ablator, heavy material and light material densities, respectively. The lengths $L_{\mathrm{abl}}, L_{\text {heav }}$ and $L_{\text {ligh }}$ correspond to the widths of the the ablator, heavy material layer and light material layer, respectively. The wit$\mathrm{dth}$ of the high pressure region is denoted by $L_{\mathrm{drv}}$ and is set to $L_{\mathrm{drv}}=40 \mu \mathrm{m}$ in all simulations. The pressure $P_{\mathrm{amb}}$ is the ambient pressure and $P_{\mathrm{drv}}$ is the drive pressure. The perturbation at the heavy/light material interface $\delta(x)$ is sinusoidal with wavelength $L_{x}$ and amplitude $A_{\mathrm{p}}$ :

$$
\delta(x)=A_{\mathrm{p}} \sin \left(2 \pi x / L_{x}\right) .
$$

See Fig. 1 for a sketch of the simulation setup.

We consider two sets of parameters, listed in Table 1: The first set (labeled "OMEGA") corresponds to the experiments performed by Kuranz et al. on the OMEGA laser [14], while the second set (labeled "NIF") corresponds to the experiment proposed by Flaig et al. [6] for the National Ignition Facility. In the OMEGA case we set the ambient pressure to a few times the atmospheric pressure, which means that we neglect any preheat effects. In the NIF case, we set the ambient pressure instead to a value that is chosen such that the initial temperature of the heavy material layer is $\sim 2800 \mathrm{~K}$, the boiling temperature of copper (see Appendix A for an estimation of preheat effects in the NIF case).

The materials assumed for the OMEGA case are polyimide $\left(\mathrm{C}_{22} \mathrm{H}_{10} \mathrm{O}_{5} \mathrm{~N}_{2}\right)$ for the heavy material (which is also the ablator) and carbonized resorcinol formaldehyde (CRF) foam for the light material [14]. In the NIF case, we assume $\mathrm{CH}$ plastic for both the ablator and the light material and copper for the heavy material [6].

A summary of the simulations presented in this paper can be found in Table 2 .

\section{Simulations with pre-imposed magnetic field}

We first consider the simple case where we solve just the ideal MHD equations (neglecting the terms on the right-hand side of Eq. (1c) and on the second line of Eq. (1d)), but with a pre-imposed uniform magnetic field. For these simulations, we use the OMEGA set of parameters (see the first three rows of Table 2 for the parameters of these simulations).

Figure 2 shows plots of the density taken at $t=21 \mathrm{~ns}$. For all

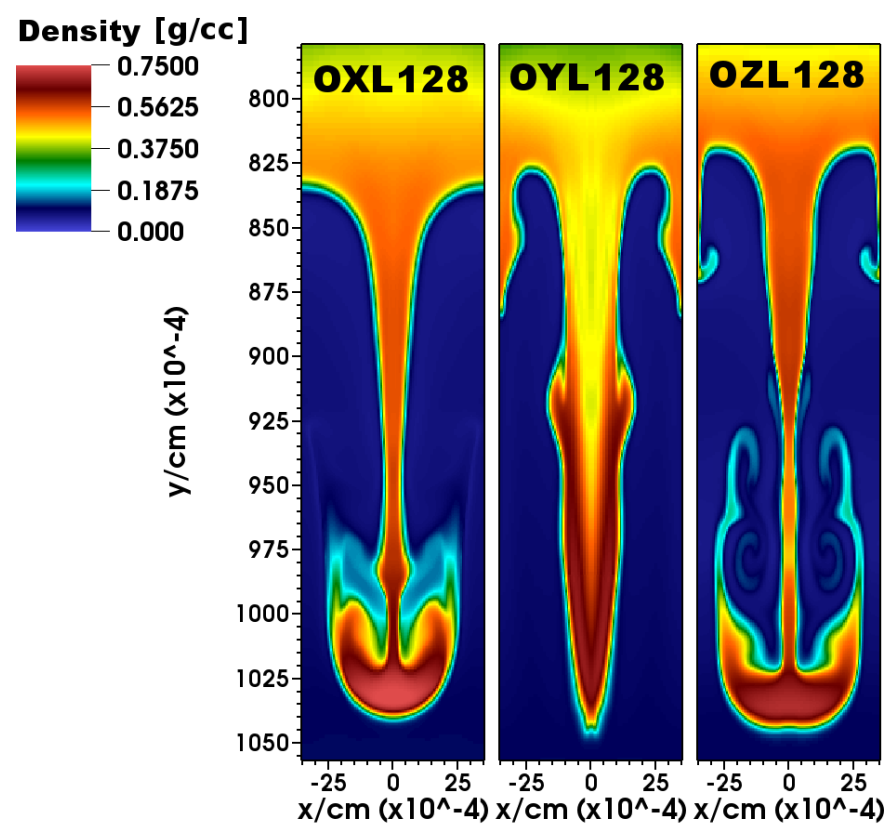

Figure 2: Plots of the density for simulations with pre-imposed magnetic field; at time $t=21 \mathrm{~ns}$. (left panel) Pre-imposed $x$-field. (middle panel) Pre-imposed $y$-field. (right panel) Pre-imposed $z$-field.

three cases, the plasma beta (not shown) varies between $\sim 10$ and $\sim 1000$ in the spike region. The impact of the magnetic field on the spike morphology is significant in the case of models OXL128 and OYL128, while in the case of model OZL128 it is small (compare with Fig. 3 (left panel) below). The spike morphology in model OYL128, with no mushroom caps at the spike tips and with bubbles and spikes having about the same width, resembles the morphology found in the Kuranz et al. experiment (cf. Fig. 3 in [14]; see also Fig. 4 in [1]).

With respect to the initial magnetic field strength, the influence on the spike morphology is strongest for model OXL128, since this model has an initial field of only $0.02 \mathrm{MG}$, which is one order of magnitude smaller than in the other two models. This is not surprising, since the field configuration in this model allows both for the amplification of the magnetic field by the non-uniform flow field, and also for the generation of a large $B_{y}$ component, and, as a consequence, for a much more complex interaction between matter and magnetic fields than in the other two models.

These results demonstrate that a magnetic field whose components lie inside the two-dimensional plane ( $B_{x}$ and $B_{y}$ components) can have a significant effect on the hydrodynamics even for relatively weak fields, while the impact of a $B_{z}$ field is small, similar to what has been found in [1].

In a two-dimensional setting like it is considered in this paper, no $B_{x}$ or $B_{y}$ components will be generated by the Biermann battery effect. Consequently, the dynamical impact of the self-generated field is expected to be small, even if fields of significant strength were to be generated. The situation will, however, be fundamentally different in a three-dimensional setting, where all three field components can be generated and new three-dimensional magnetic field effects come into play. 


\begin{tabular}{llll}
\hline \hline Parameter & Description & Value (OMEGA case) & Value (NIF case) \\
\hline$L_{x}$ & lateral domain size & $71 \mu \mathrm{m}$ & $200 \mu \mathrm{m}$ \\
$L_{y}$ & longitudinal domain size & $1420 \mu \mathrm{m}$ & $3000 \mu \mathrm{m}$ \\
$L_{\text {abl }}$ & ablator width & $0 \mu \mathrm{m}$ & $200 \mu \mathrm{m}$ \\
$L_{\text {heav }}$ & heavy layer width & $150 \mu \mathrm{m}$ & $150 \mu \mathrm{m}$ \\
$\rho_{\text {abl }}$ & ablator density & - & $1.014 \mathrm{~g} \mathrm{~cm}^{-3}$ \\
$\rho_{\text {heav }}$ & heavy material density & $1.43 \mathrm{~g} \mathrm{~cm}^{-3}$ & $8.96 \mathrm{~g} \mathrm{~cm}^{-3}$ \\
$\rho_{\text {ligh }}$ & light material density & $0.05 \mathrm{~g} \mathrm{~cm}^{-3}$ & $1.014 \mathrm{~g} \mathrm{~cm}^{-3}$ \\
$A_{\mathrm{p}}$ & perturbation amplitude & $2.5 \mu \mathrm{m}$ & $10 \mu \mathrm{m}$ \\
$P_{\text {amb }}$ & ambient pressure & $5 \cdot 10^{6} \mathrm{~g} \mathrm{~cm}^{-1} \mathrm{~s}^{-2}$ & $2.5 \cdot 10^{12} \mathrm{~g} \mathrm{~cm}^{-1} \mathrm{~s}^{-2}$ \\
$P_{\mathrm{drv}}$ & drive pressure & $8 \cdot 10^{13} \mathrm{~g} \mathrm{~cm}^{-1} \mathrm{~s}^{-2}$ & $3 \cdot 10^{14} \mathrm{~g} \mathrm{~cm}^{-1} \mathrm{~s}^{-2}$ \\
\hline
\end{tabular}

Table 1: Parameters used for the numerical simulations presented in this paper. The first column lists the symbols used in the text, and the second column describes the parameter. The third column lists the parameter values for the OMEGA case and the fourth column the values for the NIF case. In the OMEGA case, the heavy material layer is also the ablator. Note that in the NIF case, we chose a heavy layer width of $150 \mu \mathrm{m}$ instead of $200 \mu \mathrm{m}$ as proposed in [6].

\begin{tabular}{lccccllll}
\hline \hline Model & Parameter Set & $\boldsymbol{q}$ & $\boldsymbol{S}_{\mathrm{GenOhm}}$ & $\boldsymbol{R}_{u}$ & $B_{0 x}[\mathrm{MG}]$ & $B_{0 y}[\mathrm{MG}]$ & $B_{0 z}[\mathrm{MG}]$ & Numerical Resolution \\
\hline OXL128 & OMEGA & no & no & - & 0.02 & 0 & 0 & $0.55 \mu \mathrm{m}\left(=L_{x} / 128\right)$ \\
OYL128 & OMEGA & no & no & - & 0 & 0.2 & 0 & $0.55 \mu \mathrm{m}\left(=L_{x} / 128\right)$ \\
OZL128 & OMEGA & no & no & - & 0 & 0 & 0.2 & $0.55 \mu \mathrm{m}\left(=L_{x} / 128\right)$ \\
OL128 & OMEGA & no & no & - & 0 & 0 & 0 & $0.55 \mu \mathrm{m}\left(=L_{x} / 128\right)$ \\
OCL128 & OMEGA & yes & no & - & 0 & 0 & 0 & $0.55 \mu \mathrm{m}\left(=L_{x} / 128\right)$ \\
OCEL128 & OMEGA & yes & yes & no & 0 & 0 & 0 & $0.55 \mu \mathrm{m}\left(=L_{x} / 128\right)$ \\
OCERL128 & OMEGA & yes & yes & yes & 0 & 0 & 0 & $0.55 \mu \mathrm{m}\left(=L_{x} / 128\right)$ \\
NL128 & NIF & no & no & - & 0 & 0 & 0 & $2.34 \mu \mathrm{m}\left(=L_{x} / 128\right)$ \\
NCL128 & NIF & yes & no & - & 0 & 0 & 0 & $2.34 \mu \mathrm{m}\left(=L_{x} / 128\right)$ \\
NCEL128 & NIF & yes & yes & no & 0 & 0 & 0 & $2.34 \mu \mathrm{m}\left(=L_{x} / 128\right)$ \\
\hline
\end{tabular}

Table 2: Summary of the simulations presented in this paper. The first column gives the model name. The second column describes which set of parameters has been used; with "OMEGA" and "NIF" refering to the parameters in the third and fourth column of Table 1, respectively. The third, fourth and fifth columns indicate if the thermal conduction $(\boldsymbol{q})$, generalized Ohms law $\left(\boldsymbol{S}_{\text {GenOhm }}\right)$ and friction force $\left(\boldsymbol{R}_{u}\right)$ terms have been included or not, respectively. The fifth, sixth and seventh columns state the $x, y$ and $z$-components of the initial magnetic field. The eighth column lists the effective numerical resolution. 


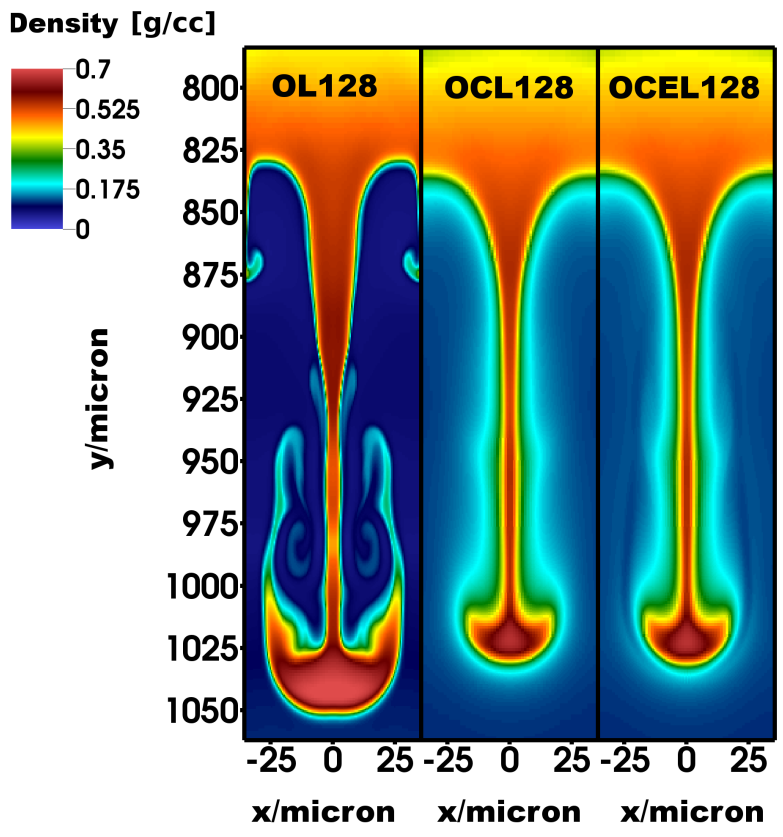

Figure 3: Plots of the density for simulations using the OMEGA set of parameters; at time $t=21 \mathrm{~ns}$. (left panel) Ideal MHD (thermal conduction and extended MHD terms neglected). (middle panel) With thermal conduction but extended MHD terms neglected. (right panel) Only friction force $\left(\boldsymbol{R}_{u}\right)$ term neglected.

\section{Simulations with self-generated magnetic fields}

\subsection{Spike morphology}

We now look at the case where the initial magnetic field is zero, but where the generalized Ohms law term $\boldsymbol{S}_{\mathrm{GenOhm}}$ is included, so that a magnetic field is generated by the Biermann battery effect. We perform simulations using the OMEGA set of parameters as well as simulations using the NIF set of parameters (Table 1). See Table 2, rows 4 to 10 for a summary of the parameters of the simulations discussed in this section.

Figure 3 shows the spike morphology after $21 \mathrm{~ns}$ for simulations using the OMEGA set of parameters. Thermal conduction has a strong effect in this regime: In the case without thermal conduction (left panel of Fig. 3), spikes with large mushroom caps are produced, while in the case with thermal conduction (middle panel of Fig. 3), mushroom caps are very small, resembling more the morphology observed by Kuranz et al. [9] in their experiment on OMEGA. In comparison to thermal conduction, the effect of self-generated magnetic fields is practically negligible (compare the middle and right panels in Fig. 3).

In the case of the NIF models (Fig. 4), thermal conduction leads to a significant broadening of the spikes (compare left and middle panels), but does not suppress the Kelvin-Helmholtz roll-up at the spike tips. The self-generated magnetic fields are even weaker than in the OMEGA case (see Fig. 5 below) and have no appreciable effect on the morphology (compare middle and right panels).

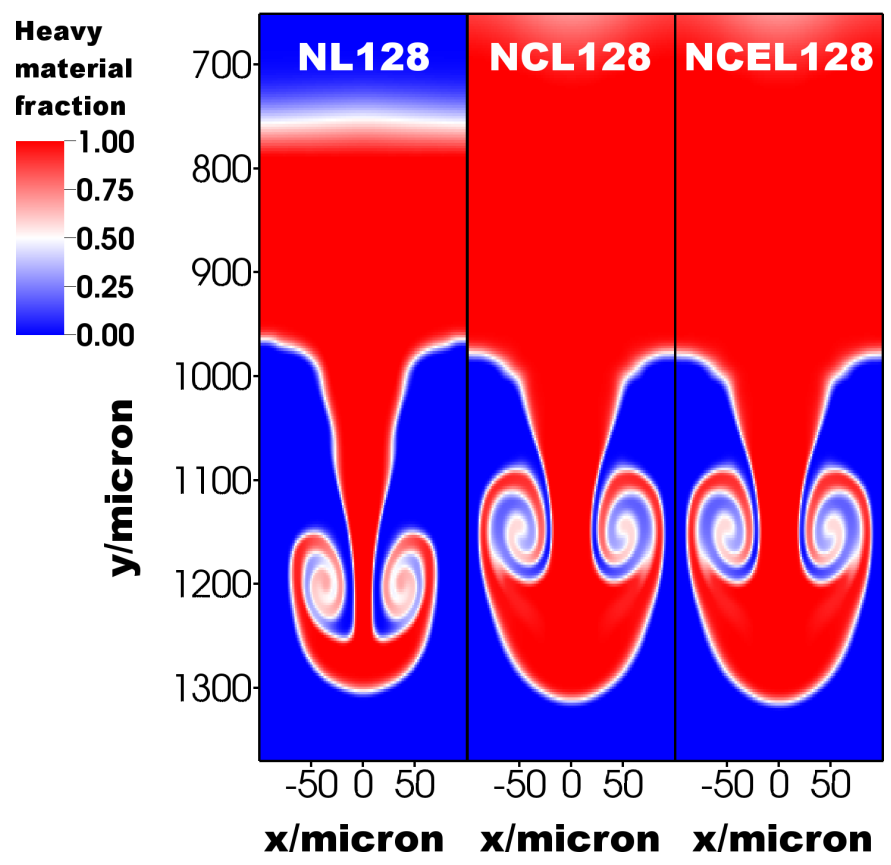

Figure 4: Plots of the heavy material fraction for simulations using the NIF set of parameters; at time $t=80 \mathrm{~ns}$. (left panel) Ideal MHD (thermal conduction and extended MHD terms neglected). (middle panel) With thermal conduction but extended MHD terms neglected. (right panel) Only friction force $\left(\boldsymbol{R}_{u}\right)$ term neglected.

\subsection{Magnetic field strength and morphology}

We now focus our attention on the magnetic field strength and morphology. Figure 5 shows the magnetic field in the spike region for the models NCEL128, OCEL128 and OCERL128. The magnetic field in the OMEGA case simulation OCEL128 is by a factor of $\sim 2$ bigger than in the NIF model NCEL128. This is not unexpected, since the density contrast is higher in the OMEGA case, leading to steeper temperature gradients and higher rates of magnetic field generation.

The plasma beta (not shown) is of order $\sim 10-100$ for model OCEL128 and $\sim 100-1000$ for model NCEL128. Note that the magnetic field obtained in model OCEL128 (which includes magnetic field dissipation due to the thermal force) is one order of magnitude smaller than the estimate given in [1], which neglected any dissipative effects.

Including the friction force term in the induction equation reduces the magnetic field by more than two orders of magnitude (compare the plots in the upper panel of Fig. 5). The plasma beta (not shown) in model OCERL128 is of order $\gtrsim 10^{5}$, and the magnetic field consequently plays no role in the RTI dynamics.

Assuming that the field strengths found in model OCERL128 can be considered representative for the case with threedimensional perturbations also, would deem it highly unlikely that magnetic fields could be the cause of the RTI spike extensions reported by Kuranz et al. [9]. Instead, our results suggest that thermal conduction is the most likely agent responsible for the featureless morphology of spikes found in this experiment. 

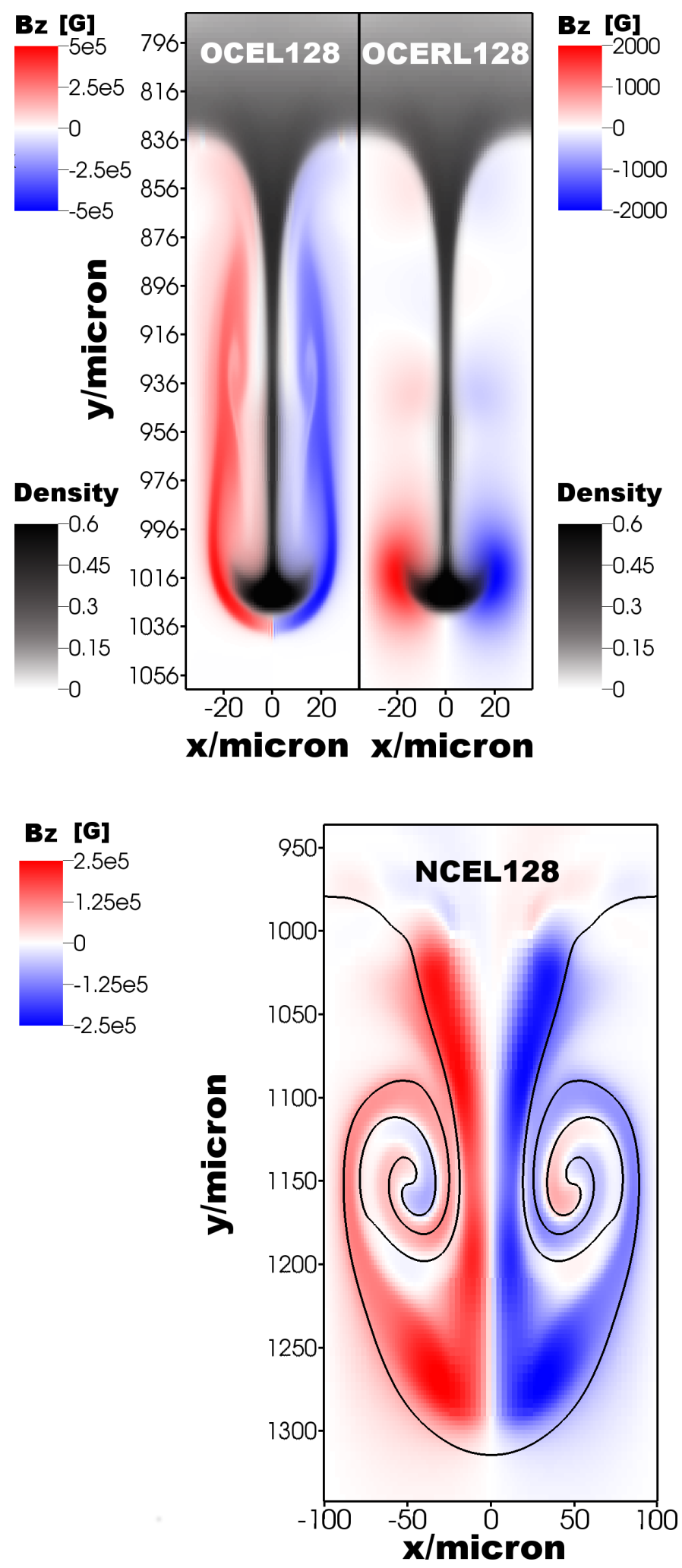

Figure 5: Magnetic field strength in Gauss for some of the simulations. (upper left panel) Model OCEL128 (OMEGA parameter set, only friction force $\left(\boldsymbol{R}_{u}\right)$

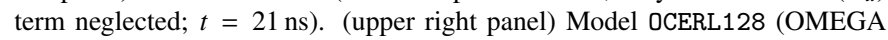
parameter set, with the friction force term included; $t=21 \mathrm{~ns}$ ). The upper two panels contain also a plot of the density (in g/cc). (lower panel) Model NCEL128 (NIF parameter set, only friction force $\left(\boldsymbol{R}_{u}\right)$ term neglected; $\left.t=80 \mathrm{~ns}\right)$. The contour line corresponds to a heavy material fraction of 0.5 .

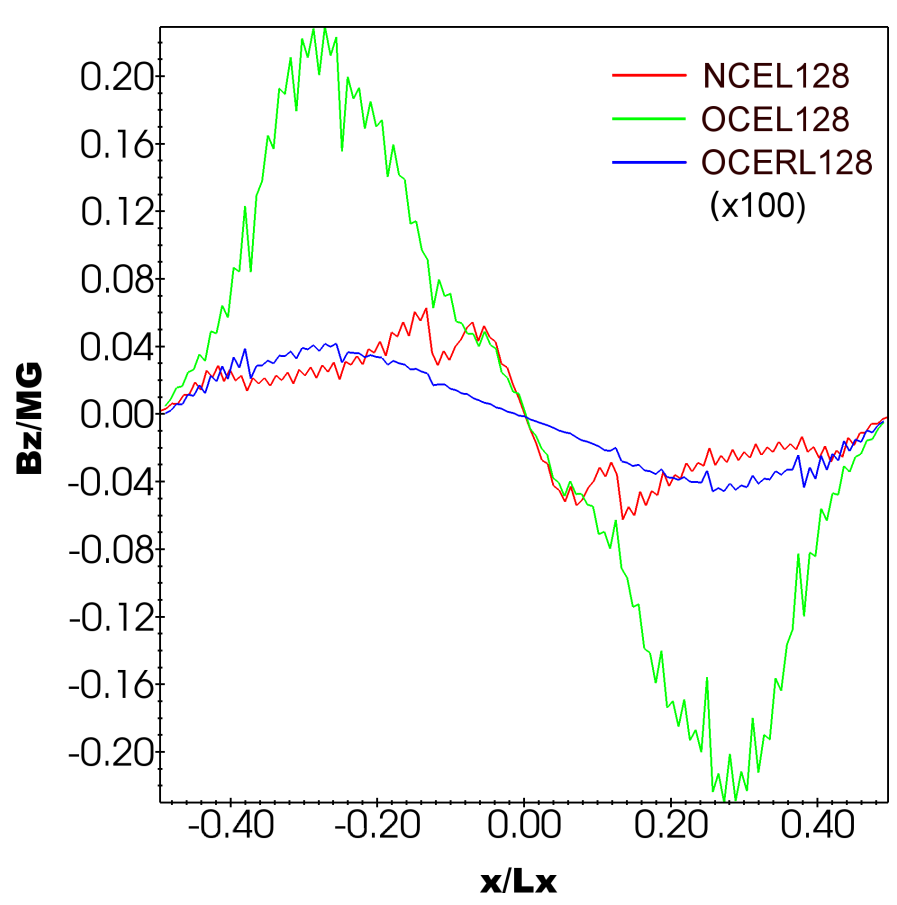

Figure 6: Magnetic field averaged over the longitudinal direction for models NCEL128, OCEL128 and OCERL128. Note that the curve corresponding to model OCERL128 has been stretched in the vertical direction by a factor of 100 .

\subsection{Measuring magnetic fields in blast-wave driven Rayleigh- Taylor experiments}

Magnetic fields in high-energy density physics experiments can be measured via monoenergetic proton radiography [23]. This technique relies on the direct proportionality between the proton deflection angle and magnetic field strenght integrated along the path of the proton (see Appendix B).

Magnetic fields have recently been measured by this technique for the first time in an ablative RTI experiment [17]. Up to now, no such measurements exist for the case of the classical RTI considered in the present paper.

Figure 6 shows the longitudinally averaged magnetic field strength for models NCEL128, OCEL128, and OCERL128. The average magnetic field is about 5 times greater in model OCEL128 than in model NCEL128. However, since the target in the OMEGA case is shorter than in the NIF case by a factor of two, the proton deflection angles will be about 2.5 times greater.

\section{Conclusions}

We studied simulations of blast-wave driven RTI including the effects of thermal conduction and self-generated magnetic fields, under conditions characteristic of high-energy density laser-driven Rayleigh-Taylor instability experiments. Our main findings and conclusions can be summarized as follows:

- In models with pre-imposed magnetic field, even relatively weak fields (plasma $\beta \sim 100$ ) can have a significant effect. Orientation of the field is a very important factor. 
- For both experimental conditions considered (OMEGA and NIF), thermal conduction has been identified as the dominant factor in determining the spike morphology. Self-generated magnetic fields are found to play only a minor role, even when the friction force term is neglected.

- Proton deflection angles are about 2.5 times greater in the OMEGA case than in the NIF case.

- Including the friction force term reduces the magnetic field strength by about two orders of magnitude, resulting in a plasma beta of $\beta \gtrsim 10^{5}$. These makes most magnetic field effects weak and difficult to diagnose. In particular, fields of this strength can not explain the morphology observed in the Kuranz et al. [14] experiment.

We note that our OMEGA models cannot be directly compared to the Kuranz et al. [14] experiment, since the models are two-dimensional. Further work will be devoted to perform three-dimensional simulations, which will allow for more complex magnetic fields, with more than one field component being generated.

\section{Acknowledgements}

MF and TP were supported by the DOE grant DE-FG5209NA29548 and the NSF grant AST-1109113. The authors want to thank Paul Keiter for his help in analyzing the preheat effects provided in Appendix Appendix A. This research used resources of the National Energy Research Scientific Computing Center, which is supported by the Office of Science of the U.S. Department of Energy under Contract No. DE-AC0205CH11231. The software used in this work was in part developed by the DOE Flash Center at the University of Chicago.

\section{Appendix A. Estimation of preheat effects}

In laser-driven shock experiments, the target may experience significant heating prior to the arrival of the shock. This effect, which is commonly referred to as "preheat", is caused by superthermal electrons and x-rays which are generated in the drive corona. Below we estimate the amount of preheat that the target would experience in the NIF case.

We assume that a fraction of $10^{-3}$ of the laser energy is converted into $\mathrm{x}$-rays and super-thermal electrons that will preheat the target [see Fig. 101.62 in 24]. Assuming $100 \mathrm{~kJ}$ of incident laser energy (Flaig et al. 2014), we estimate that $\sim 100 \mathrm{~J}$ of energy would preheat the target. Assuming further a target diameter of $2400 \mu \mathrm{m}$ (Flaig et al. 2014) and a width of the heavy material (copper) layer of $150 \mu \mathrm{m}$, we find that it would take about $86 \mathrm{~J}$ to heat the copper to its boiling point of $\sim 2800 \mathrm{~K}$. It would require an additional $278 \mathrm{~J}$ of energy to vaporize the melted copper, which greatly exceeds the estimated laser energy available for preheating the target. Consequently, we assume that in the NIF case, the heavy material layer is at a temperature of $\sim 2800 \mathrm{~K}$.

\section{Appendix B. Proton deflection angle}

The Lorentz force on a proton moving with velocity $\boldsymbol{v}=v \mathbf{e}_{y}$ in a magnetic field $\boldsymbol{B}=B \mathbf{e}_{z}$ is given by

$$
\boldsymbol{F}=q \boldsymbol{v} \times \boldsymbol{B}=e v B_{z},
$$

yielding the acceleration

$$
a_{x}=\frac{e v}{m_{\mathrm{p}}} B_{z}
$$

where $m_{\mathrm{p}}$ is the proton mass. The lateral velocity component aquired by the proton is thus

$$
v_{x}(x)=\frac{e v}{m_{\mathrm{p}}} \int_{0}^{L_{x} / v} B_{z}(x, v t) \mathrm{d} t=\frac{e v}{m_{\mathrm{p}}} \int_{0}^{L_{x}} B_{z}(x, y) \mathrm{d} y,
$$

from which the deflection angle follows as:

$$
\theta(x) \approx \frac{v_{x}}{v}=\frac{e}{m_{\mathrm{p}}} \int_{0}^{L_{x}} B_{z}(x, y) \mathrm{d} y=\frac{e L_{x}}{m_{\mathrm{p}}} \overline{B_{z}(x)} .
$$

\section{References}

[1] B. Fryxell, C. C. Kuranz, R. P. Drake, M. J. Grosskopf, A. Budde, T. Plewa, N. Hearn, J. Hansen, A. Miles, J. Knauer, HEDP 6 (2010) 162165.

[2] A. Nishiguchi, Jpn. J. Appl. Phys. 41 (2002) 326

[3] A. R. Miles, Astrophys. J. 696 (2009) 498-514.

[4] J. Rayleigh, Scientiftic Papers 2 (1900) 200.

[5] G. Taylor, Proc. R. Soc. London A 201 (1950) 192.

[6] M. Flaig, T. Plewa, P. A. Keiter, R. P. Drake, M. Grosskopf, C. Kuranz, H.-S. Park, High Energy Density Physics 12 (2014) 35-45.

[7] R. P. Drake, J. J. Carroll, III, K. Estabrook, S. G. Glendinning, B. A. Remington, R. Wallace, R. McCray, Astrophys. J. 500 (1998) L157.

[8] C. C. Kuranz, R. P. Drake, D. R. Leibrandt, E. C. Harding, H. F. Robey, A. R. Miles, B. E. Blue, J. F. Hansen, H. Louis, M. Bono, J. Knauer, D. Arnett, C. A. Meakin, Astrophys. Space Sci. 298 (2005) 9-16.

[9] C. C. Kuranz, R. P. Drake, E. C. Harding, M. J. Grosskopf, H. F. Robey, B. A. Remington, M. J. Edwards, A. R. Miles, T. S. Perry, B. E. Blue, T. Plewa, N. C. Hearn, J. P. Knauer, D. Arnett, D. R. Leibrandt, Astrophys. J. 696 (2009) 749-759.

[10] D. Ryutov, R. P. Drake, J. Kane, E. Liang, B. A. Remington, W. M. WoodVasey, Astrophys. J. 518 (1999) 821-832.

[11] J. Kane, D. Arnett, B. A. Remington, S. G. Glendinning, J. Castor, R. Wallace, A. Rubenchik, B. A. Fryxell, The Astrophysical Journal Letters 478 (1997) L75.

[12] J. Kane, D. Arnett, B. A. Remington, S. G. Glendinning, G. Bazn, E. Mller, B. A. Fryxell, R. Teyssier, The Astrophysical Journal 528 (2000) 989.

[13] C. C. Kuranz, R. P. Drake, M. J. Grosskopf, A. Budde, C. Krauland, D. C. Marion, A. J. Visco, J. R. Ditmar, H. F. Robey, B. A. Remington, A. R. Miles, A. B. R. Cooper, C. Sorce, T. Plewa, N. C. Hearn, K. L. Killebrew, J. P. Knauer, D. Arnett, T. Donajkowski, Physics of Plasmas 16 (2009) 056310.

[14] C. C. Kuranz, R. P. Drake, M. J. Grosskopf, B. Fryxell, A. Budde, J. F. Hansen, A. R. Miles, T. Plewa, N. Hearn, J. Knauer, Physics of Plasmas 17 (2010) 052709

[15] F. Modica, T. Plewa, A. Zhiglo, High Energy Density Physics 9 (2013) $767-780$.

[16] A. Budde, R. Drake, C. Kuranz, M. Grosskopf, T. Plewa, N. Hearn, HEDP 6 (2010) 135-142.

[17] Manuel, M. J.-E. and Li, C. K. and Seguin, F. H. and Frenje, J. and Casey, D. T. and Petrasso, R. D. and Hu, S. X. and Betti, R. and Hager, J. D. and Meyerhofer, D. D. and Smalyuk, V. A., Phys. Rev. Lett. 108 (2012) 255006. 
[18] L. Gao, P. M. Nilson, I. V. Igumenschev, S. X. Hu, J. R. Davies, C. Stoeckl, M. G. Haines, D. H. Froula, R. Betti, D. D. Meyerhofer, Physical Review Letters 109 (2012) 115001.

[19] S. Braginskii, Reviews of Plasma Physics 1 (1965) 205.

[20] B. Fryxell, K. Olson, F. X. Timmes, M. Zingale, D. Q. Lamb, P. MacNeice, R. Rosner, J. W. Truran, H. Tufo, Astrophys. J. Suppl. Ser. 131 (2000) 273.

[21] D. Salzmann, Oxford University Press (2006).

[22] L. Dongwook, A. E. Deane, Journal of Computational Physics 228 (2009) 952-975.

[23] C. K. Li, F. H. Seguin, J. Frenje, J. R. Rygg, R. D. Petrasso, Phys. Rev. Lett. 97 (2006) 135003

[24] S. G. Glendinning et al., Phys. Plas. 10 (2003). 